Article

\title{
Limits of Solid Solutions and Thermal Deformations in the L-Alanine-L-Serine Amino Acid System
}

\author{
Elena Kotelnikova ${ }^{1}$, Roman Sadovnichii ${ }^{1}$ (D), Lyudmila Kryuchkova ${ }^{1}$ and Heike Lorenz ${ }^{2, *(D)}$ \\ 1 Department of Crystallography, St. Petersburg State University, Universitetskaya emb. 7/9, \\ 199034 St. Petersburg, Russia; kotelnikova.45@mail.ru (E.K.); rsadovnichii@gmail.com (R.S.); \\ kryuchkova.2106@gmail.com (L.K.) \\ 2 Max Planck Institute for Dynamics of Complex Technical Systems, Sandtorstrasse 1, \\ 39106 Magdeburg, Germany \\ * Correspondence: lorenz@mpi-magdeburg.mpg.de
}

Received: 4 June 2020; Accepted: 13 July 2020; Published: 16 July 2020

\begin{abstract}
The limits of solid solutions and thermal deformations in the L-alanine-L-serine (L-ala-L-ser) amino acid system have been determined. Thirteen amino acid mixtures with various proportions of the components L-ser/L-ala were studied using powder X-ray diffraction techniques. It was found that the regions of solid solutions in the system are rather limited and cover less than $10 \mathrm{~mol} . \%$ from each component side. The thermal behavior of the components L-ser and L-ala and the composition L-ser/L-ala $=90 / 10$ were studied by temperature-resolved powder X-ray diffraction. The heating of L-ser and L-ala only causes thermal deformations, while two-phase mixtures with the 90/10 L-ser/L-ala ratio form solid solutions at elevated temperatures. Additionally, the parameters of the thermal deformation tensor for L-ser and L-ala were calculated, and the figures of their thermal expansion coefficients were plotted and analyzed. The study conducted is of high applicability, since amino acids are active components of various biological, geological, and technological processes, including those at elevated temperatures, and have numerous applications in life-science industries.
\end{abstract}

Keywords: L-serine; L-alanine; enantiomers; isomorphic miscibility; thermal expansion; PXRD; TRPXRD

\section{Introduction}

Amino acids are typical representatives of molecular crystals with chiral molecules. They are considered as some of the most actively synthesized organic compounds, since they are widely used-for example, in the pharmaceutical and food industries-and, consequently, participate in a variety of biological and technological processes taking place at different temperatures [1-3]. Amino acids are abundant in geological media, as, in contrast to proteins, they are able to survive relatively high temperatures. Being typical representatives of chiral organic compounds, they are used for determining the age of sedimentary rocks [4-6]. This method of dating is based on the capability of L- and D-enantiomers of amino acids to undergo mutual transformations leading to racemization in the scale of geological time. The above-mentioned underlines the significance of the present work, which aims to solve several fundamental and applied problems related to discovering the limits of solid solutions and the thermal behavior of the components of amino acid systems.

In the review of B. Saha [7] and our recent work [8], it was already mentioned that the number of publications reporting thermal deformations of organic crystal structures is scarce compared to the number of related investigations on inorganic compounds. At the same time, publications on thermal deformations of amino acids or chiral substances, which play a particularly important role in living matter, are even less numerous. Examples are the works from B. Nicolaï et al. [9] 
and ourselves [8,10-12]. We investigated the thermal deformations of crystal structures in the following systems: the components and two solid solutions formed in the L-threonine-L-allo-threonine diastereomer system [10], the components in the L-malic acid-D-malic acid system [8,11], and the components formed in the L-valine-L-isoleucine amino acid system [12].

L-Amino acid enantiomers are known to more frequently occur in nature and have more practical applications. This fact motivated our interest in binary systems of different amino acids with the same chirality. The reported studies of such systems are rather scarce and, according to our knowledge, include the publications $[13,14]$ and our works [15-19].

On the contrary, rather numerous are investigations into systems of different amino acids which also have different chiralities-i.e., where the amino acid molecules are D- and L-enantiomers (see, for example, the review [16]). Here, it is worth mentioning a great contribution to the investigation of racemic compositions of the above type systems made by B. Dalhus and C. Görbitz [20-23].

The present work continues our study of systems consisting of L-enantiomers of different amino acids. In the following, we report the results of (1) the limits of solid solutions formed in the L-alanine-L-serine system, and (2) the thermal deformations of crystal structures of L-alanine (L-ala) and L-serine (L-ser). The work aims at a deeper understanding of the structure-property relationships of amino acids as chiral compounds.

\section{Materials and Methods}

\subsection{Materials}

Alanine, $\mathrm{C}_{3} \mathrm{H}_{7} \mathrm{NO}_{2}$, and serine, $\mathrm{C}_{3} \mathrm{H}_{7} \mathrm{NO}_{3}$, (Figure 1) are aliphatic proteinogenic amino acids and are found in many naturally occurring proteins. Both amino acids have one chiral center and, therefore, can exist as L- and D-enantiomers. In the serine molecule (Figure 1b), the methyl $\left(\mathrm{CH}_{3}\right)$ end group in alanine (Figure 1a) is replaced by a methylene $\left(\mathrm{CH}_{2}\right)$ moiety with an $\mathrm{OH}$ group.<smiles>CC(N)C(=O)O</smiles>

(a)<smiles>NC(CO)C(=O)O</smiles>

(b)

Figure 1. Structural formulae of alanine (a) and serine (b) molecules.

The known crystal structures of both amino acids are characterized by the same space group $P 2{ }_{1} 2{ }_{1} 2_{1}$. The first results of deciphering the crystal structures of alanine and serine were reported several decades earlier [24,25]. Later, these results were refined and high-pressure data were added [26,27]. Moreover, numerous investigations included studies of the mechanical, optic, magnetic, and electron properties of alanine and serine crystals [28-32].

L-alanine and L-serine (99\% purity) were obtained from Merck, Zug, Switzerland, and were used as obtained. As a solvent, deionized water was applied.

\subsection{Methods}

Crystals in the L-alanine-L-serine system were obtained by spontaneous crystallization from the aqueous solutions of 13 different L-ala/L-ser mixtures by reducing the temperature with the subsequent evaporation of the solvent. Mixtures with different contents of the components (mol.\%) of L-ser/L-ala $=0 / 100,7 / 93,10 / 90,15 / 85,25 / 75,35 / 65,50 / 50,65 / 35,75 / 25,85 / 15,90 / 10,93 / 7$, and 100/0 were weighed on a Shimadzu AX200 scale (accuracy $0.0001 \mathrm{~g}$ ). The solutions were prepared for a saturation temperature of $50{ }^{\circ} \mathrm{C}$ with the constant stirring of the solution using a magnetic stirrer, and afterwards were filtered through a microporous filter (pore size $1.2 \mu \mathrm{m}$ ). The solution was poured into a Petri dish and kept at room temperature for 5-7 days. The evaporation rate of the solvent was regulated by changing 
the position of the lid on the Petri dish. The resulting crystalline precipitate was removed from the solution and quickly dried on filter paper.

All the 13 samples obtained were investigated by means of Powder X-ray Diffraction (PXRD) using a Rigaku MiniFlex II diffractometer (Rigaku Co., Tokyo, Japan) with $\mathrm{Cu}_{\mathrm{K} \alpha}$ and $\mathrm{Co}_{\mathrm{K} \alpha}$ irradiation and the $2 \theta$ range of $5-60^{\circ}$. Three samples (L-ala, L-ser, and a sample of composition L-ser/L-ala $=90 / 10 \mathrm{~mol} . \%$ ) were studied by Temperature-Resolved Powder X-ray Diffraction (TRPXRD). These experiments were conducted in the atmospheric air using a Rigaku Ultima IV diffractometer (Rigaku Co., Tokyo, Japan) equipped with a high-temperature accessory and the following settings: $\mathrm{Co}_{\mathrm{K} \alpha}$ irradiation, the $2 \theta$ range of $5-60^{\circ}$, and the temperature range of $23-200^{\circ} \mathrm{C}$ with the temperature pitch of $10^{\circ} \mathrm{C}$. The X-ray patterns were processed and the unit cell parameters in the whole temperature range were calculated using the PDXL 2.7 (Rigaku Co., Tokyo, Japan) and Topaz (Bruker AXS GmbH, Karlsruhe, Germany) software.

The temperature dependences of the orthorhombic unit cell parameters were used to calculate the thermal expansion coefficients (CTE) of the L-ala and L-ser crystal structures $\left(\alpha, 10^{-6}{ }^{\circ} \mathrm{C}^{-1}\right)$ along the three orthogonal axes of the thermal deformation tensor $\left(\alpha_{11}, \alpha_{22}, \alpha_{33}\right)$. The values obtained were utilized to plot the projections of the CTE figures onto the $a b, a c$, and $b c$ planes of the corresponding orthorhombic crystal structures. In the orthorhombic syngony, the directions of the tensor axes coincide with those of the crystallographic axes. The TEV software (Thomas Langreiter and Volker Kahlenberg, Institute of Mineralogy and Petrography, Innsbruck, Austria) was used to calculate the thermal deformation tensor and plot the CTE figures.

\section{Results and Discussion}

\subsection{Limits of Solid Solutions}

Relatively similar sizes and shapes of the alanine and serine molecules (Figure 2a,b) would suggest isomorphic miscibility or, in other words, the formation of solid solutions in their mixtures.

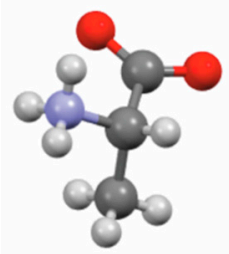

(a)

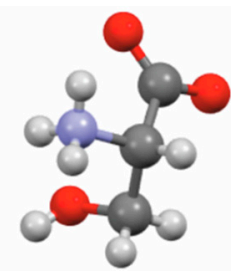

(b)

Figure 2. Molecules of alanine (a) and serine (b).

This hypothesis was totally valid in the case of the L-threonine-L-allo-threonine diastereomers with the same chirality ( $\mathrm{L}$ referring to $\mathrm{C}_{\alpha}$ ), where the authors observed the phenomenon of the continuous isomorphic miscibility of the components, which is only rarely found in chiral systems [10]. On the other hand, in the system of the L-threonine-D-threonine enantiomers [33] and the system of the D-threonine-L-allo-threonine diastereomers [34], both containing components with different chiralities, despite the identical molecular compositions and very close sizes and shapes of the molecules, the regions of solid solutions appeared to be rather limited. Three other investigated systems formed by the L-enantiomers of different amino acids-i.e., L-valine-L-isoleucine [15,16], L-valine-L-leucine [17], and L-isoleucine-L-leucine [18]—are characterized by the presence of non-equimolar discrete heterocompounds $\left(\mathrm{V}_{2} \mathrm{I}\right.$ (valine/isoleucine $\left.=2: 1\right), \mathrm{V}_{3} \mathrm{~L}$ (valine/leucine $=3: 1$ ), and $\mathrm{I}_{3} \mathrm{~L}$ (isoleucine/leucine $=3: 1$ ), respectively) and limited solid solutions in the vicinities of the heterocompounds and pure components.

As seen in Figure 3 from the X-ray patterns of the co-precipitated mixtures of L-ala and L-ser, in the L-alanine-L-serine system neither discrete heterocompounds nor solid solutions were found within the studied composition range. The $\mathrm{X}$-ray patterns shown contain two sets of peaks, one corresponding to 
L-ala and the other to L-ser. The relative intensities of each peak set vary with the changes in the mixture composition, which proves that the mixtures studied are conglomerates or just mechanical mixtures.

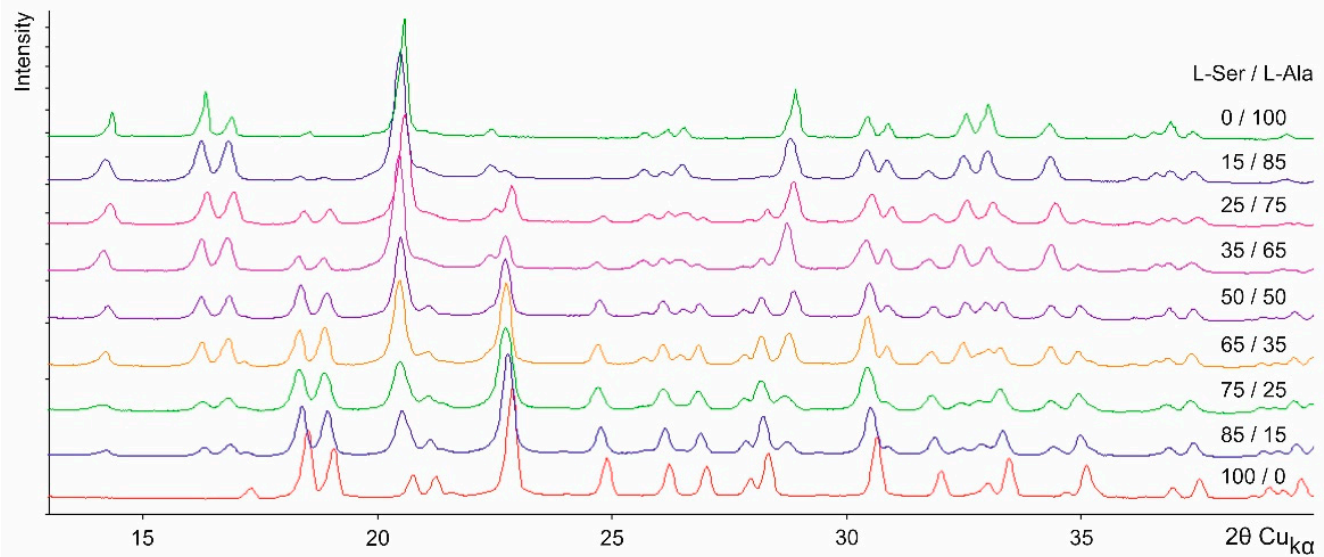

Figure 3. X-ray patterns $\left(2 \theta \mathrm{Cu}_{\mathrm{K} \alpha}\right)$ of co-precipitated mixtures (mol. \%): L-ser/L-ala = 0/100, 15/85, 25/75, 35/65, 50/50, 65/35, 75/25, 85/15, and 100/0.

In order to more precisely delineate the potential limits of the solid solutions in the L-alanine-L-serine system, several co-precipitated mixtures with compositions close to the pure components were prepared and investigated. The obtained X-ray patterns are shown in Figure 4. These experiments succeeded in revealing a limited isomorphic miscibility in the vicinity of the components. This is proved by the absence of the admixture phase peaks and the altered peak positions in the samples with L-ser/L-ala ratios of 7/93 and 93/7 compared to the corresponding positions of the L-ser and L-ala peaks. This is also reflected in the nature of changes in the respective calculated parameters and volume of the orthorhombic cells given in Table 1. A monocrystal obtained at the L-ser/L-ala ratio of 93/7 was studied by means of Single Crystal X-ray Diffractometry (SCXRD), but revealed only the presence of the L-ser phase. This fact can be explained by the low sensibility of the SCXRD method in the diagnostics of solid solutions with low contents of light molecules. The samples with L-ser/L-ala compositions of 10/90 and 90/10 (Figure 4) show the additional presence of the other solid solution and thus biphasic behavior.

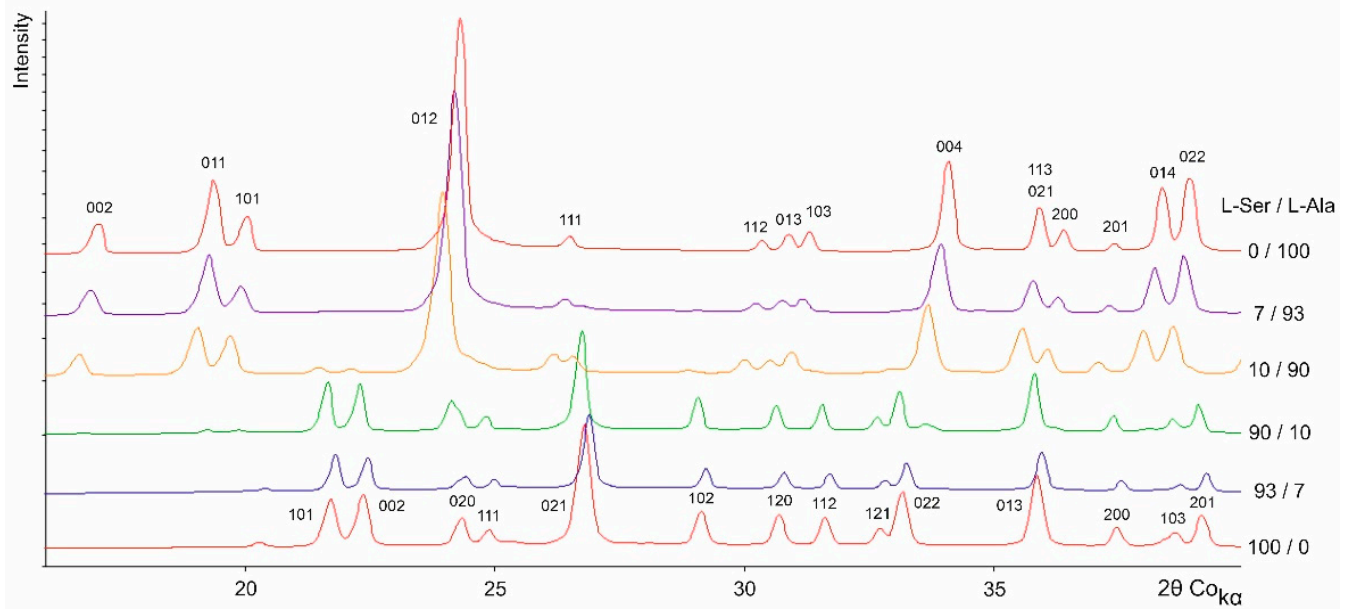

Figure 4. X-ray patterns $\left(2 \theta \mathrm{Co}_{\mathrm{K} \alpha}\right)$ of the co-precipitated mixtures (mol. \%): L-ser/L-ala = 0/100, 7/93, 10/90, 90/10, 93/7, and 100/0. 
Table 1. Parameters and volume of the orthorhombic cell for L-ser, L-ala, and their solid solutions with L-ser/L-ala ratios of 7/93 and 93/7.

\begin{tabular}{ccccc}
\hline L-Ser/L-Ala & $\boldsymbol{a}, \AA$ & $\boldsymbol{a}, \AA$ & $\boldsymbol{c}, \AA$ & $\boldsymbol{\mathbf { A }}, \AA^{\mathbf{3}}$ \\
\hline $0 / 100$ & $5.7859(5)$ & $6.0340(4)$ & $12.3441(6)$ & $430.96(5)$ \\
$7 / 93$ & $5.7908(6)$ & $6.0346(5)$ & $12.3717(8)$ & $432.34(7)$ \\
$100 / 0$ & $5.6129(3)$ & $8.5910(5)$ & $9.3456(4)$ & $450.66(4)$ \\
$93 / 7$ & $5.6096(2)$ & $8.5824(4)$ & $9.3397(3)$ & $449.65(3)$ \\
\hline
\end{tabular}

\subsection{Thermal Deformations}

Figures 5 and 6 show the X-ray patterns of L-ala and L-ser, respectively, registered at various temperatures using the TRPXRD method. According to the data obtained, in the temperature range of 23-200 ${ }^{\circ} \mathrm{C}$, L-serine and L-alanine do not undergo any polymorph transformations. Both components are exposed to thermal deformations manifested as various shifts of the peaks either towards low or high $2 \theta$ values depending on the $h k l$ indices of the peaks. It should be noted that a small splitting of the unambiguously indexed peak 020 in Figure 6, which is present at lower temperatures, is most likely caused by texture effects.

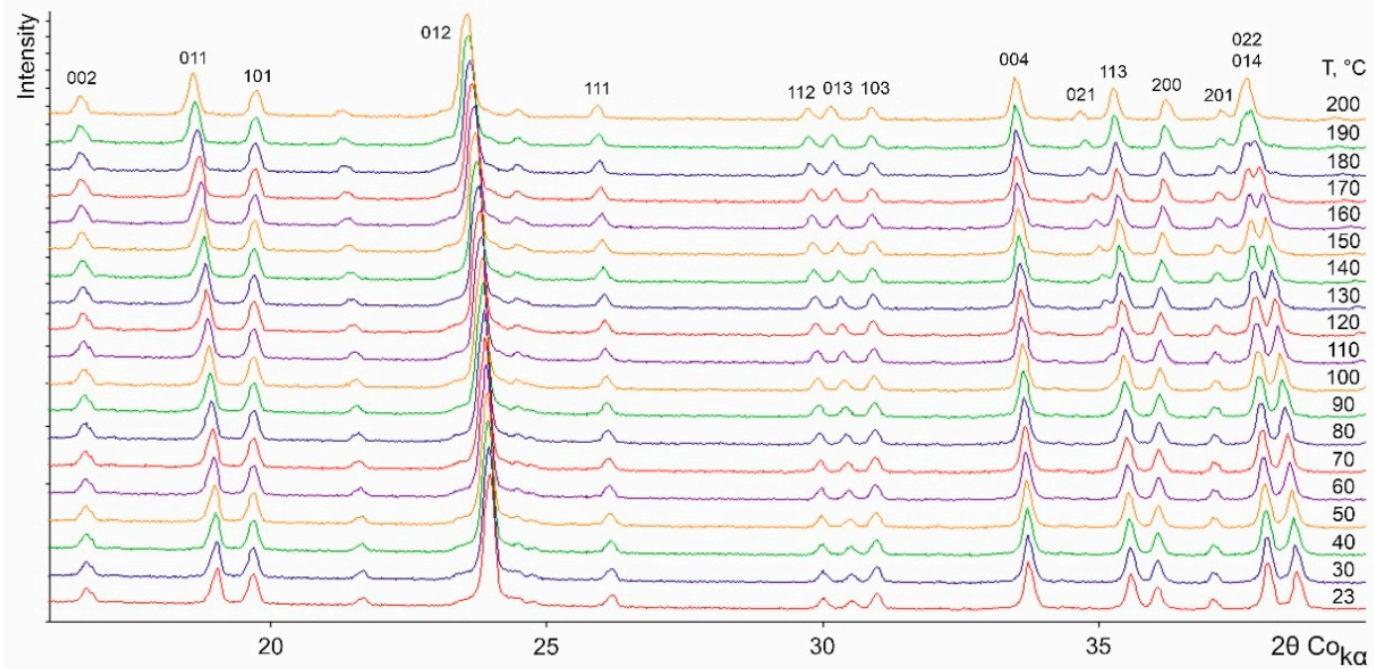

Figure 5. X-ray patterns $\left(2 \theta \mathrm{Co}_{\mathrm{K} \alpha}\right)$ of an L-ala sample obtained at various temperatures.

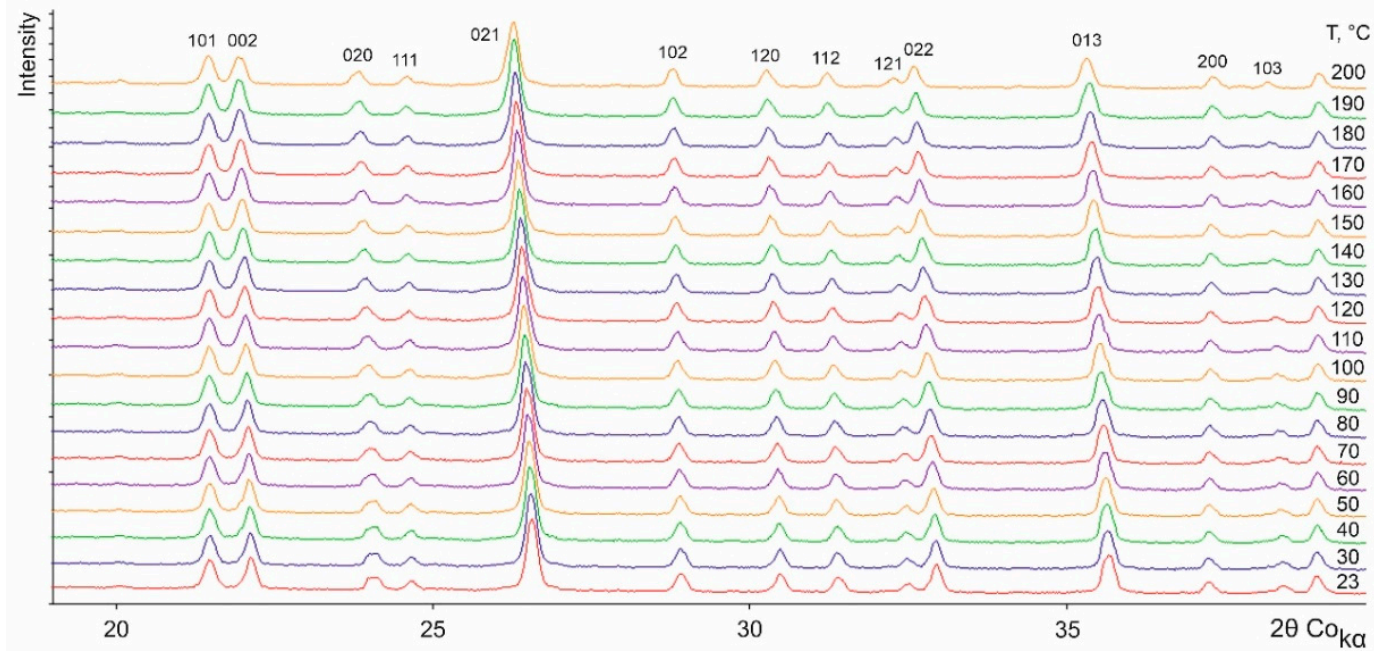

Figure 6. X-ray patterns $\left(2 \theta \mathrm{Co}_{\mathrm{K} \alpha}\right)$ of an L-ser sample obtained at various temperatures. 
Figure 7 presents a cutout of the temperature-resolved X-ray patterns of a sample with the $\mathrm{L}$-ser/L-ala ratio of 90/10 studied in a temperature range between 25 and $210{ }^{\circ} \mathrm{C}$. Initially, the sample was clearly a physical mixture of L-ser- and L-ala-rich solid solutions (as already mentioned in Figure 4). Despite a very low L-ala content, the existence of a two-phase mixture is obvious in the X-ray pattern by the presence of the 012 peak, as the most intensive peak of the L-ala phase. This peak is located close to the 020 peak of the L-ser phase (see the arrows in Figure 7). As the temperature rises, both peaks shift towards low $2 \theta$ values, with the shift of the 012 Ala peak being greater than that of the 020 Ser peak. Furthermore, the intensity of the 012 Ala peak gradually decreases until it disappears at $175^{\circ} \mathrm{C}$, while the intensity of the 020 Ser peak slightly increases, despite the fact that the sublimation point of L-ala $\left(315^{\circ} \mathrm{C}\right)$ significantly exceeds that of L-ser $\left(228^{\circ} \mathrm{C}\right)$. This could be a result of an increase in the isomorphic miscibility of L-ser and L-ala molecules when the temperature is close to $175^{\circ} \mathrm{C}$. This deems probable if one takes into account the following considerations: (1) the mixture with a similar composition of L-ser/L-ala $=93 / 07$ was shown to form a solid solution at room temperature (see Figure 4), and (2) elevated temperatures usually cause the limits of solid solutions to widen.

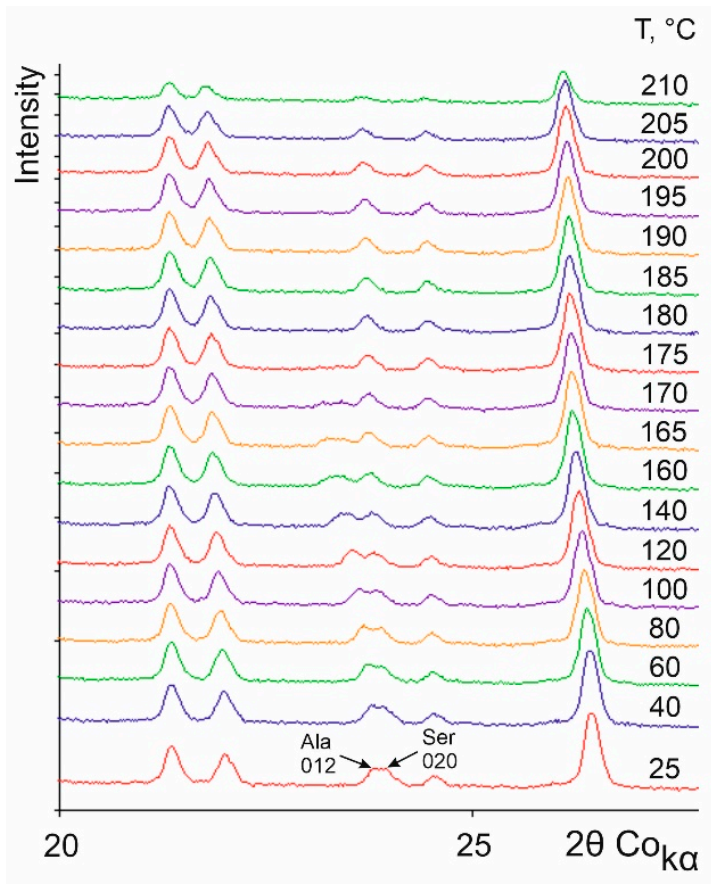

Figure 7. Fragments of the X-ray pattern $\left(2 \theta \mathrm{Co}_{\mathrm{K} \alpha}=20-28^{\circ}\right)$ of a sample with the L-ser/L-ala ratio of $90 / 10$ registered at different temperatures.

Figure 8 shows changes in the orthorhombic cell parameters and volume $V$ of L-ala and L-ser versus temperature. As the temperature increases, the $a$ parameter decreases, while the $b$ and $c$ parameters, as well as the $V$ volume of the orthorhombic cells of L-ala and L-ser, increase. Thereby, the changes of both parameters and volume are more pronounced in the unit cell of L-ala than for L-ser. The functions plotted allowed to estimate the thermal expansion coefficients (CTE) of the volumes $(\alpha \mathrm{v})$ of the corresponding orthorhombic cells. It is to be mentioned that the $\alpha_{\mathrm{v}}=170.8 \times 10^{-6}{ }^{\circ} \mathrm{C}^{-1}$ obtained for L-ala almost by half exceeds the corresponding value calculated for L-ser, $\alpha_{\mathrm{v}}=113.2 \times 10^{-6}{ }^{\circ} \mathrm{C}^{-1}$. 

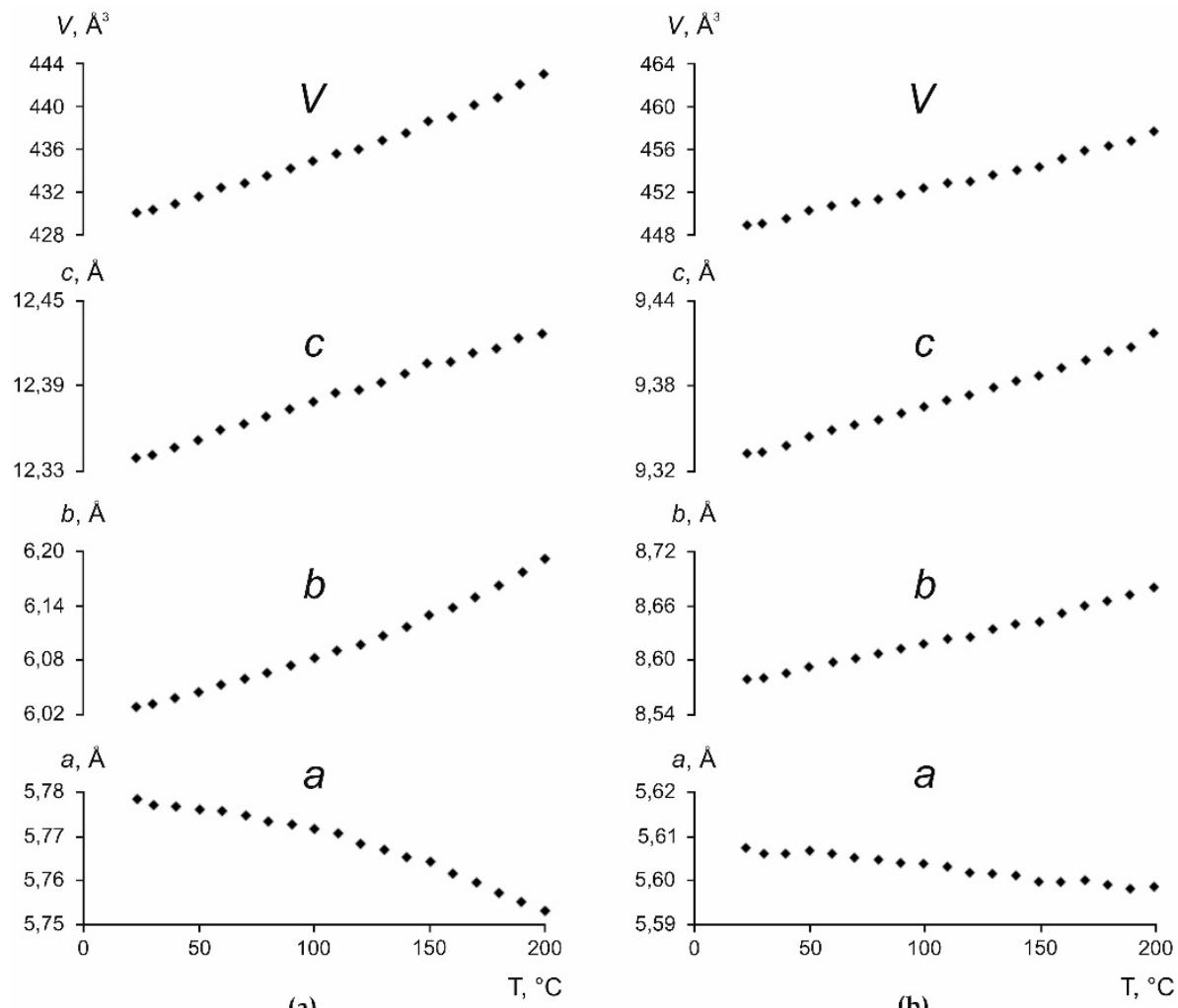

(a)

(b)

Figure 8. Changes in orthorhombic cell parameters $a, b$, and $c(\AA)$ and volume $V\left(\AA^{3}\right)$ versus the temperature for (a) L-ala and (b) L-ser.

The temperature dependences of the orthorhombic cell parameters and volume $V$ were approximated by polynomials of the first and second order. These data were used to calculate the parameters of the thermal deformation tensor and the coefficients of thermal expansion along the crystallographic axes of L-ala and L-ser, which are summarized in Table 2.

Table 2. Thermal expansion coefficients $\left(\alpha \times 10^{-6}{ }^{\circ} \mathrm{C}^{-1}\right)$ of the L-ala and L-ser orthorhombic crystal structures along the axes of the thermal deformation tensor: $\alpha_{11}=\alpha_{a}, \alpha_{22}=\alpha_{b}$, and $\alpha_{33}=\alpha_{c}$.

\begin{tabular}{cccccccccc}
\hline \multirow{2}{*}{ Amino Acid } & \multicolumn{3}{c}{$\mathbf{2 3}{ }^{\circ} \mathbf{C}$} & \multicolumn{3}{c}{$\mathbf{1 0 0}{ }^{\circ} \mathbf{C}$} & \multicolumn{3}{c}{$\mathbf{2 0 0}{ }^{\circ} \mathbf{C}$} \\
\cline { 2 - 11 } & $\boldsymbol{\alpha}_{a}$ & $\boldsymbol{\alpha}_{\boldsymbol{b}}$ & $\boldsymbol{\alpha}_{\boldsymbol{c}}$ & $\boldsymbol{\alpha}_{\boldsymbol{a}}$ & $\boldsymbol{\alpha}_{\boldsymbol{b}}$ & $\boldsymbol{\alpha}_{\boldsymbol{c}}$ & $\boldsymbol{\alpha}_{\boldsymbol{a}}$ & $\boldsymbol{\alpha}_{\boldsymbol{b}}$ & $\boldsymbol{\alpha}_{\boldsymbol{c}}$ \\
\hline L-alanine & -6.7 & 79.3 & 41 & -22.1 & 139.2 & 40.9 & -42.2 & 213.9 & 40.7 \\
L-serine & -9.6 & 52.6 & 40.7 & -9.6 & 64.1 & 48.2 & -9.6 & 78.7 & 57.7 \\
\hline
\end{tabular}

The data represented in this table, in turn, were used for plotting the figures of the thermal expansion coefficients (CTE) for L-ala (Figure 9) and L-ser (Figure 10). For better understanding, the figures also show projections of the figures onto the $a b, a c$, and $b c$ planes of the corresponding crystal structures. 


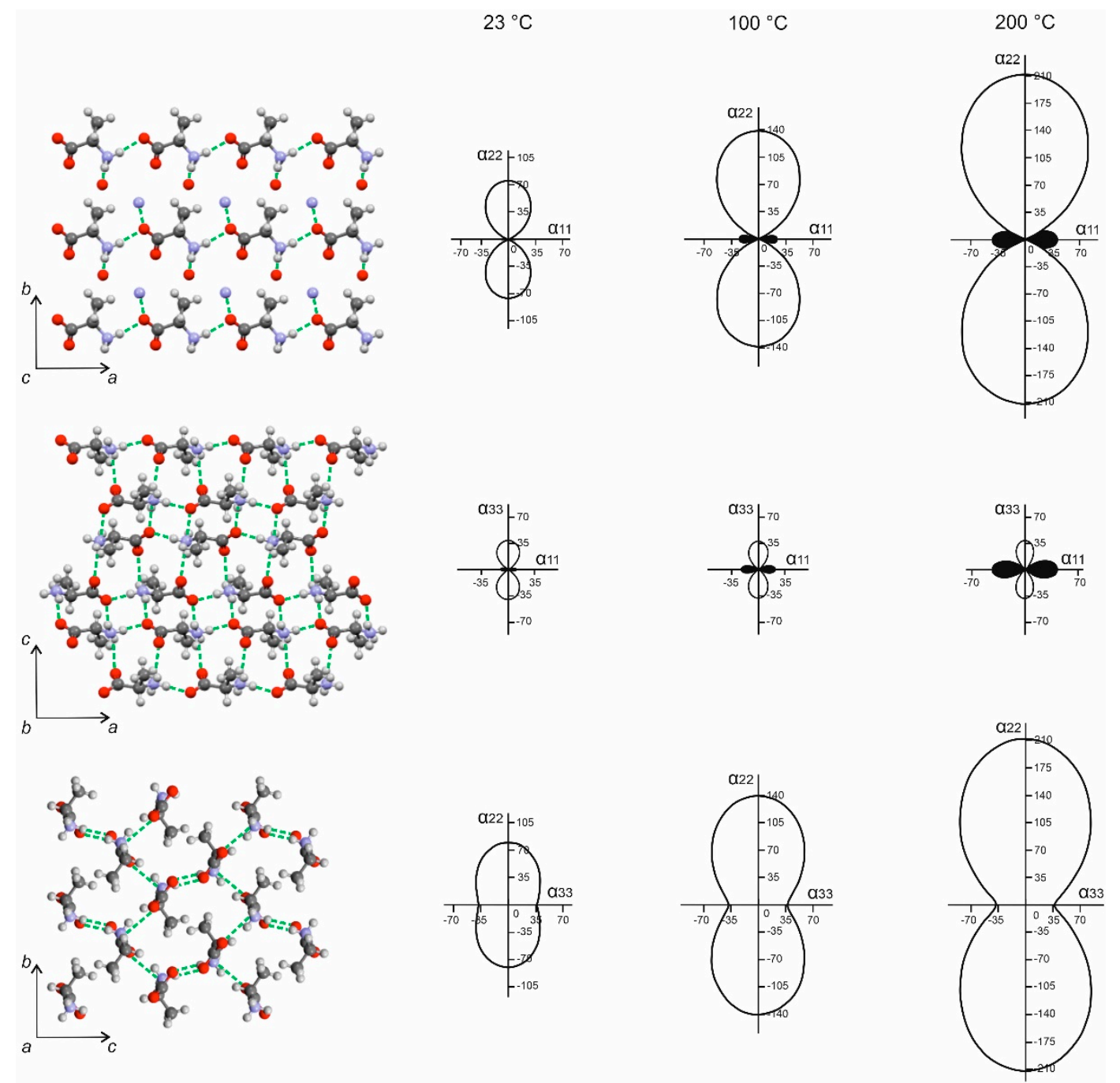

Figure 9. Projections of the figures of the thermal expansion coefficients (CTE) onto the $a b$, $a c$, and $b c$ planes of the L-ala orthorhombic cell. The CTE figures are plotted for the temperatures of 23, 100, and $200{ }^{\circ} \mathrm{C}$. Hydrogen bonds are shown as dashed lines. The projections of the orthorhombic cell are plotted using the structural data from CSD (identifier LALANINE54) [35].

An examination of the CTE figures for three different temperature conditions reveals that the thermal deformations of the crystal structures of both L-ala and L-ser are distinctly anisotropic. Both the crystal structures demonstrate a noticeable thermal expansion along the crystallographic axes $b$ and $c$ and a very significant negative (anomalous) thermal expansion (more precisely, contraction) in the direction of the $a$ axis. The crystal structures of the amino acids L-ala and L-ser can be considered as frame structures containing relatively large cavities bound by hydrogen and Van der Waals contacts. When heated, the so-called "hinge mechanism" is realized [36,37]. Its most important feature is the synchronous change of two linear parameters of the unit cell in opposite directions with the "neutral" behavior of the third parameter and the volume. In the present case, both amino acids show a multidirectional synchronous change in the parameters $a$ and $b$, as the hinges can be considered the "frames" of molecules connected by hydrogen and Van der Waals bonds. When heated, some of the atoms move away from each other, which leads to the convergence of the other part of the atoms with each other. In this case, one or another specific anisotropy is caused by the different geometries and concentrations of the hydrogen bonds of the $\mathrm{N}-\mathrm{H} \ldots \mathrm{O}$ and $\mathrm{O}-\mathrm{H} \ldots$ O type in the crystal structures of the acids. At the same time, despite some similarities, the thermal deformations of L-ala and L-ser 
have some individual patterns. In this connection, the differences of the two amino acid molecules with the different end groups (L-ala: $\mathrm{CH}_{3}$ group; L-ser: $\mathrm{CH}_{2} \mathrm{OH}$ group) should be mentioned.
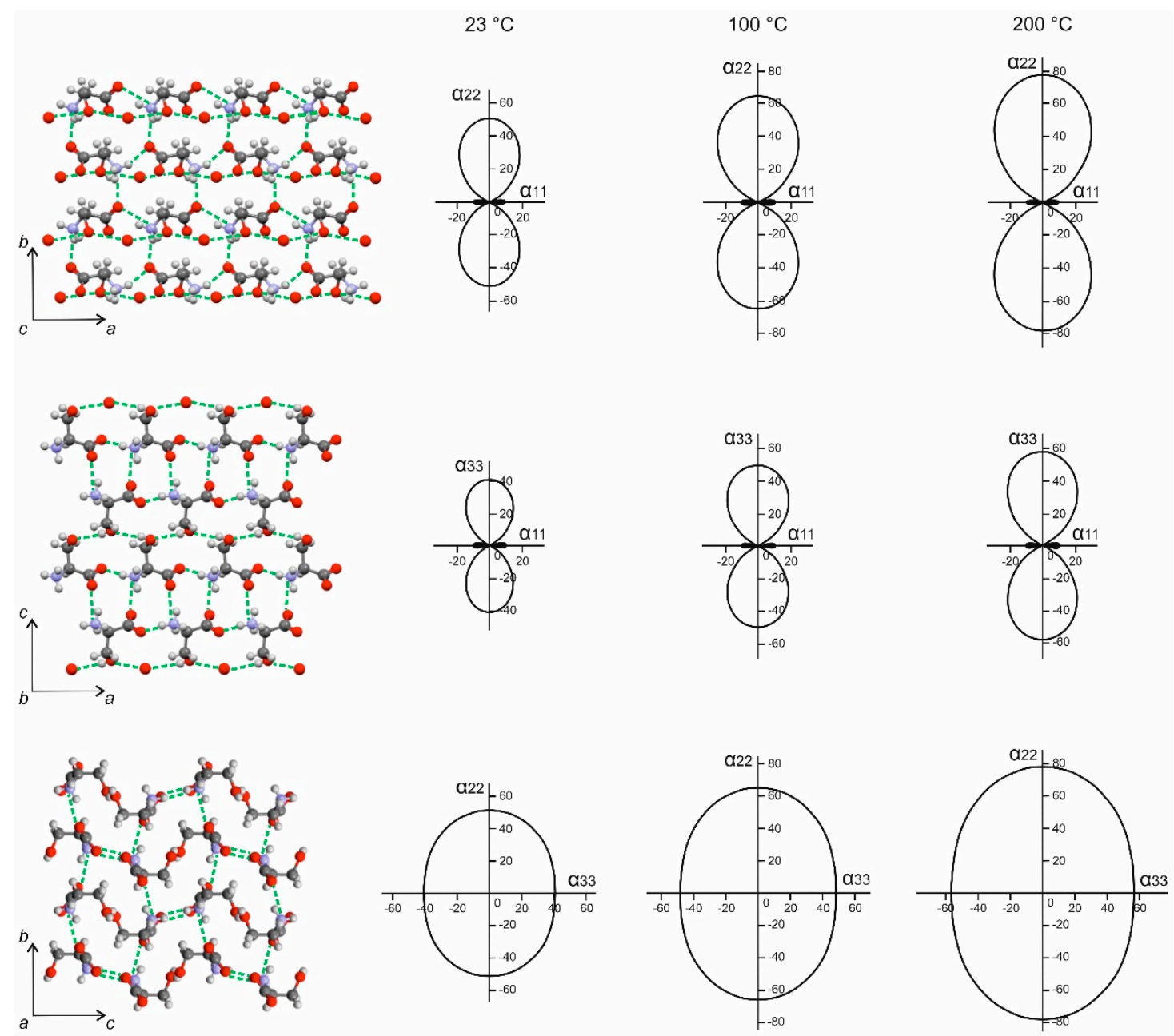

Figure 10. Projections of the figures of the thermal expansion coefficients (CTE) onto the $a b$, $a c$, and $b c$ planes of the L-ser orthorhombic cell. The CTE figures are plotted for the temperatures of 23, 100, and $200{ }^{\circ} \mathrm{C}$. Hydrogen bonds are shown as dashed lines. The projections of the orthorhombic cell are plotted using the structural data from CSD (identifier LSERIN41) [35].

L-alanine (Figure 9, Table 2): For this amino acid, the rise of temperature results in a very considerable increase in the thermal expansion coefficients in the direction of the $b$ axis, while they only slightly change in the $c$ direction. In the $a$ direction, the negative thermal expansion (contraction) coefficient increases noticeably. As a whole, the thermal deformation anisotropy increases with the elevation of temperature, but this process follows different patterns depending on the projection onto the $a b, a c$, and $b c$ crystal planes.

In the projection onto the $a b$ plane, the L-ala molecules are interconnected with the $\mathrm{N}-\mathrm{H} \ldots \mathrm{O}$ bonds to form chains positioned along the $a$ direction. In the opposite direction (in the direction of the $b$ axis), the contacts are much weaker due to their Van der Waals nature. Consequently, the maximum thermal expansion is observed along the $b$ axis and the negative thermal expansion takes place along the $a$ axis. As seen from the projection onto the $a c$ plane, the hydrogen bonds of the $\mathrm{N}-\mathrm{H} \ldots \mathrm{O}$ type exist in the direction of both $a$ and $c$ axes, but their geometries and concentrations differ depending on the particular direction and, therefore, the hydrogen contacts have different strengths. In a virtual "competition" between the hydrogen bonds, those positioned along the $c$ axis are the weakest, and so this direction is characterized by a relatively low thermal expansion, while along the $a$ axis the structure 
contracts. The same reasoning can be followed to examine thermal expansion anisotropy in the projection onto the $b c$ plane.

L-serine (Figure 10, Table 2): The elevation of temperature results in an increase in the thermal expansion coefficients in the direction of the $b$ axis, but to a lesser extent in comparison with the L-ala molecule. Along the $c$ axis, the thermal expansion coefficient becomes noticeably greater, while the negative thermal expansion coefficient in the direction of the $a$ axis does not change. This is the principal difference between the thermal deformations in L-ser and L-ala. The anisotropy of the thermal deformations in L-ser can be observed to have various manifestations in the projections onto the $a b, a c$, and $b c$ crystal planes.

In the direction of the $a$ axis, the thermal expansion is negative (anomalous) due to the presence of strong hydrogen bonds of the $\mathrm{N}-\mathrm{H} \ldots \mathrm{O}$ and $\mathrm{O}-\mathrm{H} \ldots \mathrm{O}$ types along this direction. In the $b c$ plane, the influence of the $\mathrm{N}-\mathrm{H}$... O hydrogen bonds is reinforced by the Van der Waals contacts made by the methylene $\left(\mathrm{CH}_{2}\right)$ groups. In the neighboring molecules, these groups are directed towards each other. As a result, the crystal structure undergoes thermal expansion in the directions of the crystallographic axes $b$ and $c$. It is interesting to note that in this plane the thermal deformation anisotropy was not observed and, consequently, was not affected by alterations of temperature.

\subsection{Discussion}

In one of our works [18] we reviewed some particularities of crystal structures of several (but not all) proteinogenic amino acids to estimate their abilities to form heteromolecular discrete compounds consisting of molecules with different and the same chirality. It was stated that these amino acids were prone to form dimers with the molecules connected to each other by hydrogen bonds. Such dimeric molecules, in turn, are mutually interconnected via Van der Waals bonds. This arrangement is exemplified in Figure 11a, which depicts projections of the L-valine crystal structure onto the ac plane. It is distinctly seen that the dimer molecules form layers separated by Van der Waals contacts-i.e., the structure as a whole can be regarded as layered.

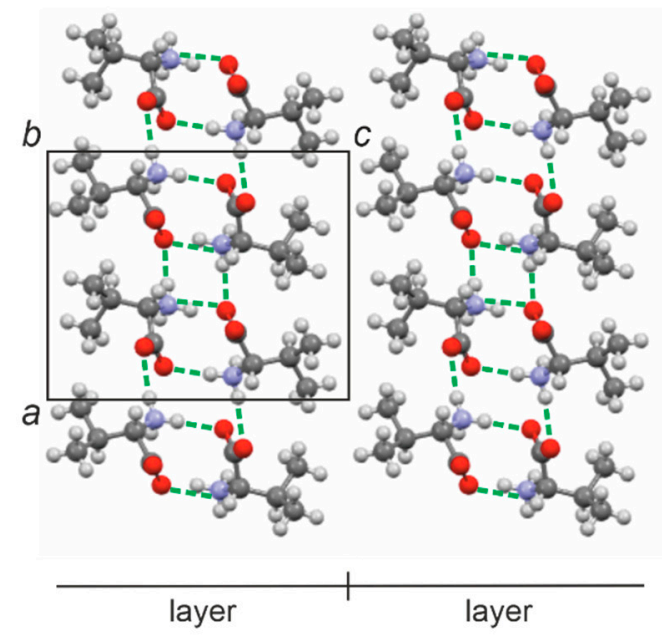

(a)

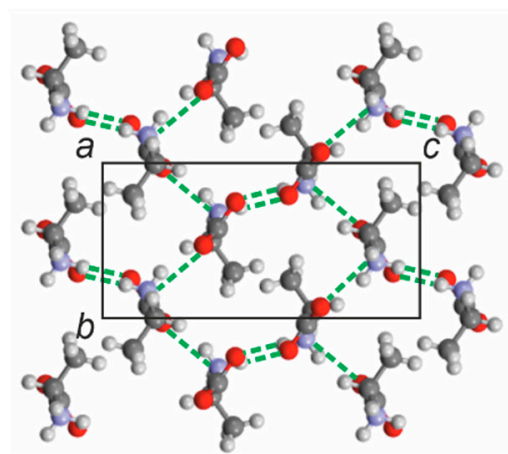

(b)

Figure 11. Projection of the L-valine crystal structure onto the ac plane of the monoclinic cell [18] (a) and projection of the L-alanine crystal structure onto the $b c$ plane of the orthorhombic cell (b). Dotted lines are hydrogen bonds. The projections of the orthorhombic cell are plotted using the structural data from CSD (identifiers LVALIN01 and LALANINE54 [35]).

Both alanine and valine molecules (Figure 12a,b) contain a methyl $\left(\mathrm{CH}_{3}\right)$ end group. However, alanine contains only one methyl on its end, while valine possesses two of them. At the first glance, this difference seems to be insignificant, but nevertheless it results in two amino acids with totally 
different crystal arrangements. While the crystal structure consisting of the dimeric molecule layers is quite viable for L-val (Figure 11a), this is not possible for L-ala.

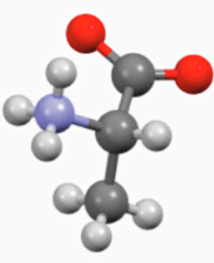

(a)

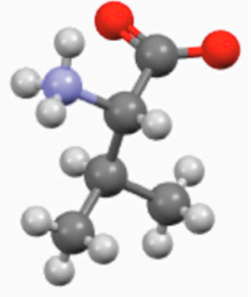

(b)

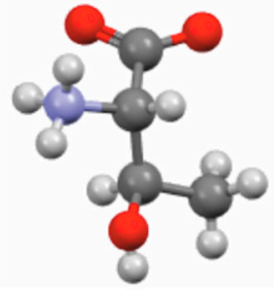

(c)

Figure 12. Molecules of alanine (a), valine (b), and threonine (c).

Figure $11 \mathrm{~b}$ shows the projection of the L-ala crystal structure onto the $b c$ plane. It can be seen that there certainly are Van der Waals contacts in the direction of the $b$ axis, but each of them is alternated with an $\mathrm{N}-\mathrm{H}$... O hydrogen bond that results in a much more robust connection. At the same time, in the direction of the $c$ axis there are two hydrogen bonds of the above type for every one of the Van der Waals bonds. Therefore, L-ala forms a "network" crystal structure (Figure 11b), similar to those of L-ser (Figure 10) and L-threonine (L-thr) [10], despite the fact that L-ser has a $\mathrm{CH}_{2} \mathrm{OH}$ end group (see Figure 2b) and L-thr possesses $\mathrm{CH}_{3}$ and $\mathrm{OH}$ end groups (Figure 12c). Furthermore, the acids L-ala, L-ser, and L-thr have another structural feature in common - that is, they all crystallize in the orthorhombic space group $P 2{ }_{1} 2_{1} 2_{1}$.

The strong anisotropy of the crystal structure, which also resulted in a negative thermal expansion (contraction in the direction of the $a$ axis), was observed as well in the related studies of L-threonine and L-allo-threonine [10]. A hinge mechanism can also be used to describe the thermal deformations of these diastereomers. Therefore, each one of L-ala, L-ser, L-thr and L-allo-thr has an orthorhombic crystal structure, while the L-valine, L-isoleucine, and L-leucine examined earlier [15-19] crystallize in monoclinic syngony.

Technically, the thermal deformations of the acids of both types ("layered" and "network") are similar to some extent. In both cases, the maximum and minimum (including "negative") thermal expansion is observed in the direction of the weakest and strongest intermolecular contacts, respectively. However, as deducible from changes of the monoclinic angle $\beta$ at elevated temperatures, the leading role in structural deformations of monoclinic crystal structures belongs to shear deformations.

The observed response of the network orthorhombic structures to rising the temperature allowed to suspect a correlation between the resulting deformation and the range of intermolecular distance variation (chiefly Van der Waals contacts) as a result of deformation of the voids (channels) via a hinged mechanism in the corresponding structures (see, for example, Figures 9 and 10).

The thermal deformations of the crystal structures of L-ala and L-ser discussed in the present article are anisotropic. However, the anisotropy of the L-ala crystals is more evident in comparison with that observed in L-ser which can be caused by the greater concentration of the Van der Waals bonds in the structure of the former amino acid. The same phenomenon can account for significant differences in the changes of the parameters and volume of their orthorhombic cells and hence corresponding thermal expansion coefficients. Here, it is worth noting again that the $\alpha_{\mathrm{v}}$ value for L-ala is almost by a half greater than that of L-ser.

Obviously, the differences in the size and shape of L-ala and L-ser molecules play only a minor role in imposing considerable limitations on the isomorphic miscibility in the L-ala-L-ser system. A much greater part is played by the differences in the nature of the intermolecular contacts in the respective amino acids' structures-namely, by a significantly greater concentration of Van der Waals bonds in the crystal structure of L-ala compared to L-ser. 


\section{Conclusions}

It was established that the L-alanine-L-serine system belongs to systems featuring eutectic behavior with very limited solid solutions (less than $10 \mathrm{~mol} \%$ ) in the vicinity of the components. Solid solutions shown are the compositions (mol.\%): L-ser/L-ala $=93 / 7$ and 7/93. Using the physical mixture L-ser/L-ala $=90 / 10$ as an example, it is demonstrated that the limits of solid solutions expand with increasing temperature. At a temperature of $175^{\circ} \mathrm{C}$, the aforementioned mixture is homogenized.

The heating of the system components L-ser and L-ala revealed a pronounced anisotropy of the thermal deformations of their crystal structures. This is reflected in the values of the parameters of the thermal deformation tensor of L-ala and L-ser and, respectively, in the figures of their thermal expansion coefficients. A multidirectional synchronous change in the CTE values with an intensive increase in the parameter $b$ and a decrease in the parameter $a$ (up to negative values) indicates a hinge mechanism of thermal deformations.

The results obtained add new insights in the structure-property relationships of amino acid systems as compounds of biological, geochemical, and industrial importance.

Author Contributions: Conceptualization, E.K. and H.L.; methodology, E.K., H.L., L.K. and R.S.; formal analysis, R.S. and L.K.; investigation, R.S., L.K. and E.K.; writing-original draft preparation, E.K.; writing-review and editing, E.K., H.L. and R.S.; visualization, E.K., R.S., L.K.; supervision, E.K. and H.L. All authors have read and agreed to the published version of the manuscript.

Funding: This research was supported by the President of Russian Federation grant to leading scientific schools (NSh-2526.2020.5).

Acknowledgments: The investigations were performed using equipment of the Resource Centre "Centre for X-ray Diffraction Studies" of St. Petersburg State University and support of the MPI Magdeburg PCF lab. The authors thank M. G. Krzhizhanovskaya, A. I. Isakov and A. A. Zolotarev Jr. for collaboration.

Conflicts of Interest: The authors declare no conflict of interest.

\section{References}

1. Giron, D. Polymorphism in the pharmaceutical industry. Therm. Anal. Calorim. 2001, 64, 37-60. [CrossRef]

2. Murakami, H. From Racemates to Single Enantiomers-Chiral Synthetic Drugs over the last 20 year. Top. Curr. Chem. 2006, 269, 273-299.

3. Bredikhin, A.A.; Bredikhina, Z.A.; Zakharychev, D. Crystallization of chiral compounds: Thermodynamical, structural and practical aspects. Mendeleev Commun. 2012, 22, 171-180. [CrossRef]

4. Robins, J.; Jones, M.; Matisoo-Smith, E. Amino Acid Racemization Dating in New Zealand: An Overview and Bibliography; Auckland Univ.: Auckland, New Zealand, 2010.

5. Killops, S. Introduction to Organic Geochemistry, 2nd edn (paperback). Geofluids 2005, 5, 236-237. [CrossRef]

6. Torres, T.; Ortiz, J.; Arribas, I.; Delgado, A.; Julià, R.; Rubí, J.A.M. Geochemistry of Persististrombus latus Gmelin from the Pleistocene Iberian Mediterranean realm. Lethaia 2009, 43, 149-163. [CrossRef]

7. Saha, B.K. Thermal Expansion in Organic Crystals. J. Indian Inst. Sci. 2017, 97, 177-191. [CrossRef]

8. Kotelnikova, E.N.; Isakov, A.; Lorenz, H. Thermal deformations of crystal structures formed in the systems of malic acid enantiomers and l-valine-l-isoleucine enantiomers. CrystEngComm 2018, 20, 2562-2572. [CrossRef]

9. Nicolaï, B.; Barrio, M.; Tamarit, J.-L.; Céolin, R.; Rietveld, I.B. Thermal expansion of L-ascorbic acid. Eur. Phys. J. Spéc. Top. 2017, 226, 905-912. [CrossRef]

10. Taratin, N.; Lorenz, H.; Binev, D.; Seidel-Morgenstern, A.; Kotelnikova, E. Solubility Equilibria and Crystallographic Characterization of the 1-Threonine/l-allo-Threonine System, Part 2: Crystallographic Characterization of Solid Solutions in the Threonine Diastereomeric System. Cryst. Growth Des. 2015, 15, 137-144. [CrossRef]

11. Isakov, A.; Kotelnikova, E.N.; Kryuchkova, L.Y.; Lorenz, H. Effect of crystallization conditions on polymorphic diversity of malic acid RS-Racemate. Trans. Tianjin Univ. 2013, 19, 86-91. [CrossRef]

12. Isakov, A.; Kotelnikova, E.; Bocharov, S.; Zolotarev, A., Jr.; Lorenz, H. Thermal deformations of the crystal structures of L-valine, L-isoleucine and discrete compound V2I. In Proceedings of the 23rd International Workshop on Industrial Crystallization (BIWIC-2016), Magdeburg, Germany, 6-8 September 2016; pp. 7-12. 
13. Koolman, H.C.; Rousseau, R.W. Effects of isomorphic compounds on the purity and morphology of L-isoleucine crystals. Aiche J. 1996, 42, 147-153. [CrossRef]

14. Kurosawa, I.; Teja, A.; Rousseau, R. Solubility measurements in the l-Isoleucine + 1-Valine + Water System at 298 K. Eng. Chem. Res. 2005, 44, 3284-3288. [CrossRef]

15. Isakov, A.; Kotelnikova, E.; Muenzberg, S.; Bocharov, S.; Lorenz, H. Solid Phases in the System L-Valine-L-Isoleucine. Cryst. Growth Des. 2016, 16, 2653-2661. [CrossRef]

16. Kotelnikova, E.; Isakov, A.; Lorenz, H. Non-equimolar discrete compounds in binary chiral systems of organic substances (Highlight). Cryst. Eng. Comm. 2017, 19, 1851-1869. [CrossRef]

17. Kryuchkova, L.; Kotelnikova, E.; Zolotarev, A., Jr.; Lorenz, H. Limits of solid solutions in the L-leucine-isoleucine system according to PXRD and SCXRD data. In Proceedings of the 25rd International Workshop on Industrial Crystallization (BIWIC-2018), Rouen, France, 5-7 September 2018; pp. 225-226.

18. Isakov, A.I.; Lorenz, H.; Zolotarev, A.A.; Kotelnikova, E.N. Heteromolecular compounds in binary systems of amino acids with opposite and same chiralities. CrystEngComm 2020, 22, 986-997. [CrossRef]

19. Kotelnikova, E.; Isakov, A.; Kryuchkova, L.; Zolotarev, A., Jr.; Bocharov, S.; Lorenz, H. Acids with Chiral Molecules as Essential Organic Compounds of Biogenic-Abiogenic Systems. In Processes and Phenomena on the Boundary between Biogenic and Abiogenic Nature; Frank-Kamenetskaya, O.V., Vlasov, D., Panova, E.G., Lessovaia, S.N., Eds.; Springer Nature Switzerland: Berlin, Germany, 2020; Volume 37, pp. 695-719.

20. Dalhus, B.; Görbitz, C.H. Molecular aggregation in crystalline 1:1 complexes of hydrophobic D- and L-amino acids. Acta Crystallogr. Sect. B Struct. Sci. 1999, 55, 424-431. [CrossRef]

21. Dalhus, B.; Görbitz, C. Molecular aggregation in selected crystalline 1:1 complexes of hydrophobic D- and L-amino acids. II. The D-norleucine series. Acta Crystallogr. 1999, C55, 1105-1112.

22. Dalhus, B.; Görbitz, C.H. Molecular aggregation in selected crystalline 1:1 complexes of hydrophobic D- and L-amino acids. III. The L-leucine and L-valine series. Acta Crystallogr. 1999, C55, 1547-1555.

23. Görbitz, C.H. Crystal structures of amino acids: From bond lengths in glycine to metal complexes and high-pressure polymorphs. Crystallogr. Rev. 2015, 21, 1-53. [CrossRef]

24. Simpson, H.J.; Marsh, R.E. The crystal structure of L -alanine. Acta Crystallogr. 1966, 20, 550-555. [CrossRef]

25. Kistenmacher, T.J.; Rand, G.A.; Marsh, R.E. Refinements of the crystal structures of DL-serine and anhydrous L-serine. Acta Crystallogr. Sect. B Struct. Crystallogr. Cryst. Chem. 1974, 30, 2573-2578. [CrossRef]

26. Moggach, S.A.; Allan, D.R.; Morrison, C.A.; Parsons, S.; Sawyer, L. Effect of pressure on the crystal structure of L-serine-I and the crystal structure of L-serine-II at 5.4 GPa. Acta Crystallogr. Sect. B Struct. Sci. 2005, 61, 58-68. [CrossRef] [PubMed]

27. Zakharov, B.; Kolesov, B.A.; Boldyreva, E.V. Effect of pressure on crystalline L- and DL-serine: Revisited by a combined single-crystal X-ray diffraction at a laboratory source and polarized Raman spectroscopy study. Acta Crystallogr. Sect. B Struct. Sci. 2012, 68, 275-286. [CrossRef] [PubMed]

28. Kimura, F.; Oshima, W.; Matsumoto, H.; Uekusa, H.; Aburaya, K.; Maeyama, M.; Kimura, F. Single crystal structure analysis via magnetically oriented microcrystal arrays. CrystEngComm 2014, 16, 6630-6634. [CrossRef]

29. Costa, S.N.; Sales, F.A.M.; Freire, V.N.; Maia, F.F.; Caetano, E.; Ladeira, L.O.; Albuquerque, E.L.; Fulco, U.L. 1-Serine Anhydrous Crystals: Structural, Electronic, and Optical Properties by First-Principles Calculations, and Optical Absorption Measurement. Cryst. Growth Des. 2013, 13, 2793-2802. [CrossRef]

30. Rajesh, K.; Kumar, P.P. Structural, Linear, and Nonlinear Optical and Mechanical Properties of New Organic L-Serine Crystal. J. Mater. 2014, 2014, 1-5. [CrossRef]

31. Podder, J.; Akhtar, F. A Study on Growth, Structural, Optical and Electrical Characterization of L-alanine Single Crystal for Optoelectronic Devices. Res. J. Phys. 2012, 6, 31-40. [CrossRef]

32. Vijayan, N.; Rajasekaran, S.; Bhagavannarayana, G.; Babu, R.R.; Gopalakrishnan, R.; Palanichamy, M.; Ramasamy, P. Growth and Characterization of Nonlinear Optical Amino Acid Single Crystal: L-Alanine. Cryst. Growth Des. 2006, 6, 2441-2445. [CrossRef]

33. Sapoundjiev, D.; Lorenz, H.; Seidel-Morgenstern, A. Solubility of Chiral Threonine Species in Water/Ethanol Mixtures. J. Chem. Eng. Data 2006, 51, 1562-1566. [CrossRef]

34. Taratin, N.; Binev, D.; Lorenz, H.; Seidel-Morgenstern, A.; Kotelnikova, E. Characterization and limits of solid solutions in binary systems of threonine diastereomers according to X-ray diffraction data. In Proceedings of the III International Conference Crystallogenesis and Mineralogy, Novosibirsk, Russia, 27 September-1 October 2013; pp. 341-I-342-II. 
35. CSD files (identifiers): LALANINE54 (L-alanine), LSERIN41 (L-serine) and LVALIN01 (L-valine). Available online: https://www.ccdc.cam.ac.uk (accessed on 16 July 2020).

36. Filatov, S.; Bubnova, R. The nature of special points on unit cell parameters temperature dependences for crystal substances. Z. Kristallogr. 2007, 26, 447-452. [CrossRef]

37. Filatov, S.; Krivovichev, S.; Bubnova, R. General Crystal Chemistry; Publishing House of St. Petersburg University: St. Petersburg, Russia, 2018; pp. 222-224. 